\title{
58. Jahrestagung der ÖGKJ in Innsbruck
}

\author{
Pilotprojekt „Abhaltung als Präsenzveranstaltung" erfolgreich \\ abgeschlossen.
}

Bereits bei der Planung der heurigen Jahrestagung war klar, dass diese aufgrund der Rahmenbedingungen ganz anders sein würde als vorhergehende.

Wir haben im Frühjahr als Tagungsorganisation mehrfach die Frage diskutiert, ob wir die Tagung durchführen oder absagen sollen.

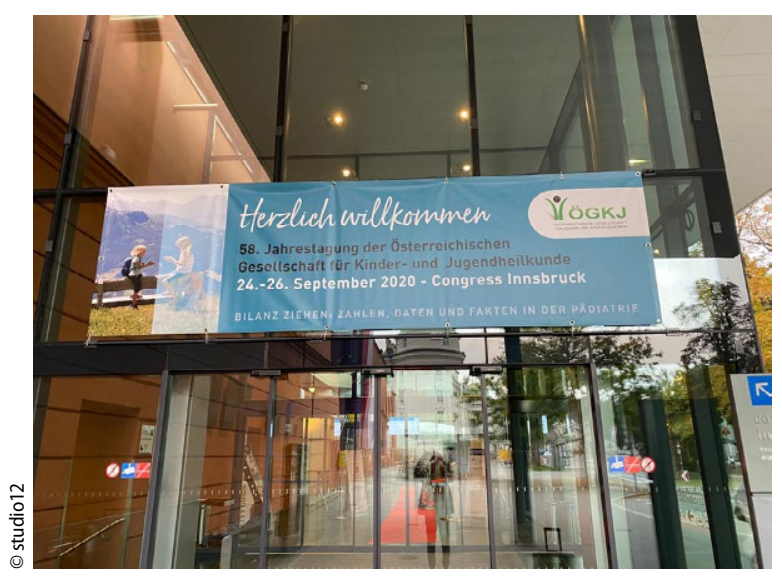

Abb. 1 ॥ Über dem Haupteingang des Kongresshauses in Innsbruck angebrachtes „Herzlich willkommen“ Transparent zur 58. ÖGKJ Jahrestagung in Innsbruck

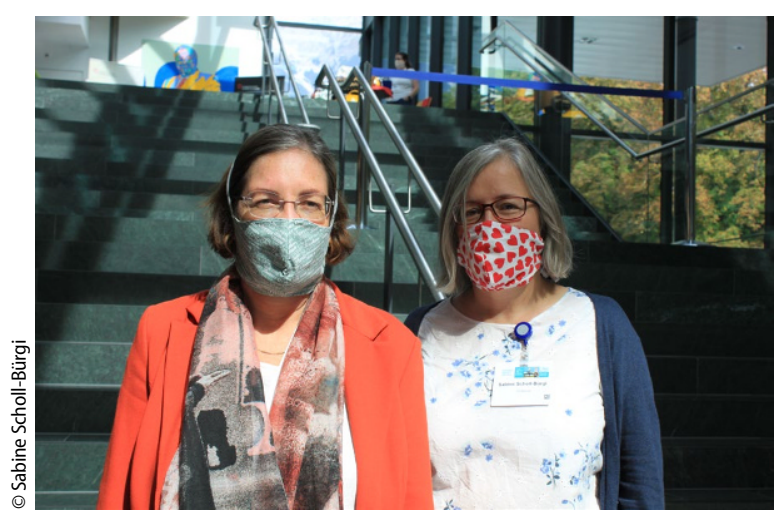

Abb. $2 \Delta$ Tagungsorganisatorinnen Daniela Karall, ÖGKJ Präsidentin, und Sabine Scholl-Bürgi, ÖGKJ Erste Sekretärin, beide Innsbruck, auf der Treppe des Kongresshauses mit von Mitarbeitern der Tiroler Hospizgemeinschaft genähten Stoffmasken
Schließlich haben wir uns mit der Unterstützung der Leitung der Klinik für Pädiatrie in Innsbruck, an der wir beide beschäftigt sind, des ÖGKJ Präsidiums, der lokalen Behörden und des Bundesministeriums für Gesundheit (BM Rudolf Anschober) dafür entschieden, die Jahrestagung vom 24.09. bis 26.09.2020 im Kongresshaus in Innsbruck als Präsenzveranstaltung abzuhalten (• Abb. 1).

Unserer Entscheidung lag folgende Philosophie zugrunde:

Wir wollten die Jahrestagung soweit möglich als Präsenzveranstaltung durchführen - selbstverständlich jeweils im Rahmen der aktuell geltenden behördlichen Vorgaben,

- weil das Leben mittelfristig mit und trotz Corona weiterhin stattfinden muss,

- weil wir als Medizinerinnen und Mediziner diejenige Gruppe sind, die prädestiniert ist, vorzuzeigen, wie gedeihliches Miteinander (gerade auch bei Veranstaltungen) gelingen kann,

- weil virtuelle Veranstaltungen nur sehr bedingt die Verpflichtung zu Fortbildung und fachlich persönlichem Austausch abdecken können,

- weil wir sie als ein positives Beispiel eines Pilotprojekts gesehen haben,

- weil wir ein positives Zeichen setzen wollten.

Die Jahrestagung hat nach aktuellem Stand der Verordnungen und der Überprüfung des Congress Innsbruck stattgefunden. Für die Jahrestagung lag bereits seit längerer Zeit ein 40 seitiges COVID-19 Präventionskon- zept vor, das erfolgreich umgesetzt wurde (Kontrolle der Anwesenden im Kongresshaus, Einhalten der Maßnahmen (Abstand, Händehygiene, Mund-Nasen-Schutz)). Mit der erwarteten Anzahl der Teilnehmerinnen und Teilnehmer befanden wir uns im behördlich vorgesehenen Rahmen (s. - Abb. 2, 3 und 4). Auf den üblicherweise am Abend des 2 . Tages stattfindenden Gesellschaftsabend wurde u. a. verzichtet.

Bis zu Beginn der Jahrestagung haben wir uns täglich an die Gegebenheiten angepasst und die Information jeweils auf der Jahrestagungs-Homepage eingefügt (www.oegkj-jahrestagung.at). Dabei ist es uns entgegengekommen, dass wir die Tagung bereits im Vorfeld als Green Event konzipiert hatten, so dass Änderungen im Programm wesentlich einfacher waren, weil z.B. das Programm online und nicht als Papierdruck vorlag. Für die Teilnehmerinnen und Teilnehmer hatten wir von zwei Mitarbeitern der Tiroler Hospizgemeinschaft 500 Stoff-

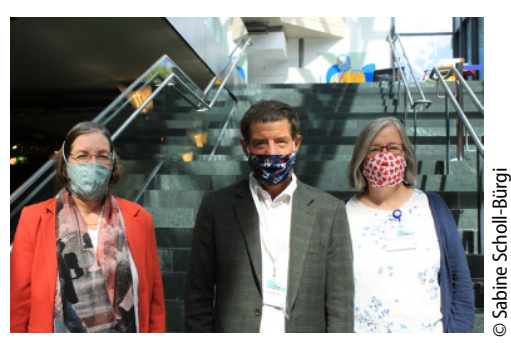

Abb. 3 ^ Tagungsorganisatorinnen Daniela Karall, ÖGKJ Präsidentin, und Sabine Scholl-Bürgi, ÖGKJ Erste Sekretärin, beide Innsbruck, mit Reinhold Kerbl, ÖGKJ Generalsekretär, auf der Treppe des Kongresshauses mit von Mitarbeitern der Tiroler Hospizgemeinschaft genähten Stoffmasken 


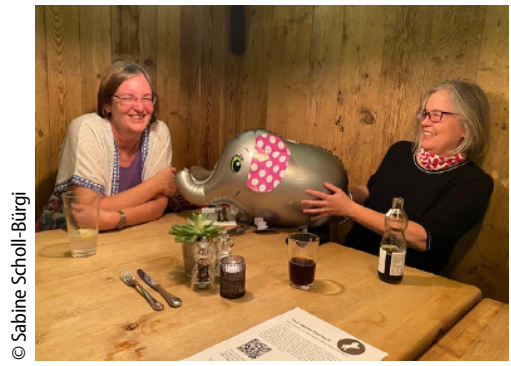

Abb. 4 \ Tagungsorganisatorinnen Daniela Karall, ÖGKJ Präsidentin, und Sabine Scholl-Bürgi, ÖGKJ Erste Sekretärin, beide Innsbruck, bei der Präsidiumssitzung am Vorabend der Jahrestagung mit Babyelefant, einem Mitbringsel von Reinhold Kerbl zur Jahrestagung

masken nähen lassen (s. • Abb. 2 und 3).

Für alle jene, die absagen (mussten), war ein Live Stream der Plenarsitzungen vorhanden. Sie bekamen den Link auf Rückmeldung zugesandt.

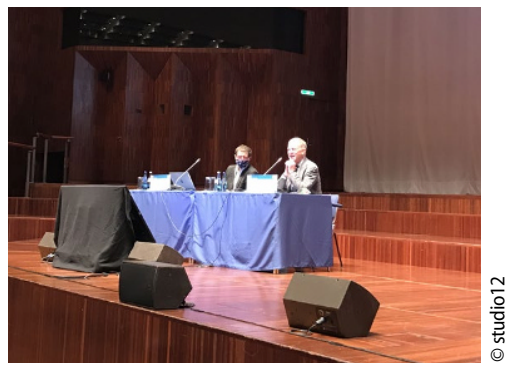

Abb. 5 \ Vorsitzende der ersten Plenarsitzung der 58. ÖGKJ Jahrestagung im Saal Tirol, Kongresshaus Innsbruck, am 24.09.2020: Prof. Dr. Reinhold Kerbl, ÖGKJ Generalsekretär, Leoben, und Prof. Dr. Wolfgang Sperl, ÖGKJ Vizepräsident, Salzburg

Trotz aller guten Vorbereitung ist es bis zuletzt sehr spannend geblieben!

Am 24.09.2020 fanden bereits einige Sitzungen statt (Vorstandssitzung, Arbeitsgruppen und Referate), die teilweise als Hybrid-Sessions (Online-Zuschaltung der nicht anwe- senden Kollegen möglich). Mittags starteten die Lunch Symposien, auch diese waren z.T. als Hybrid-Sessions aufgesetzt.

Um 14.00 fand die erste Plenarsitzung statt (s. - Abb. 5) und daran anschließend die Eröffnung (• Abb. 6), in derem Rahmen wir u.a. eine Videobotschaft von Bundesminister für Gesundheit Rudolf Anschober und eine Begrüßung durch den Tiroler Landesrat für Gesundheit DI Bernhard Tilg eingebaut hatten. In Rahmen der Eröffnung fanden heuer auch die Preisverleihungen der wissenschaftlichen Preise durch Prof. Dr. Thomas Lion, Leiter der AG Wissenschaft und Forschung, und die Verleihung der August von Reuss Medaille an Fr. MR Dr. Manuela Baumgartner statt, für die Sabine Scholl-Bürgi die Laudatio gehalten hat. Am Ende stellte Thomas Beddies die erstmals in Österreich statt-

Hier steht eine Anzeige. 
findende Ausstellung „Im Gedenken der Kinder" vor. Sie konnte während des gesamten Kongresses besichtigt werden und blieb noch weitere

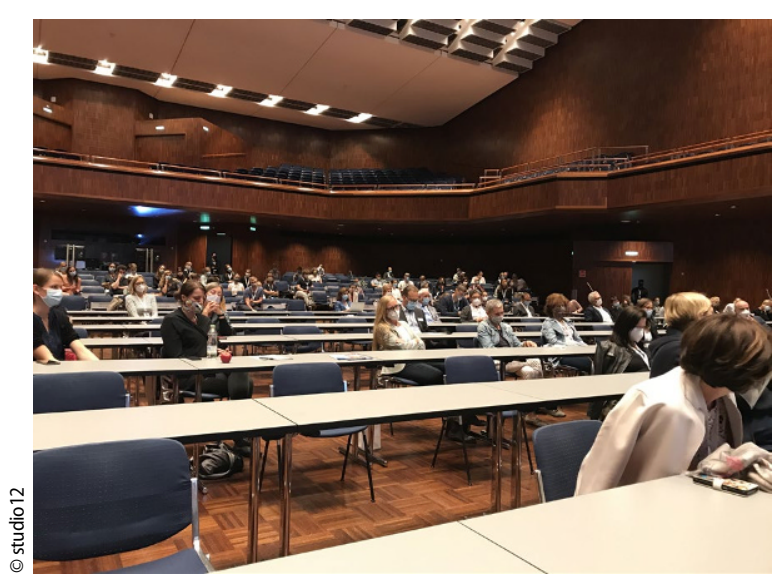

Abb. 6 ^ Plenarsitzung Saal Tirol, Kongresshaus Innsbruck, zur Eröffnung der 58. ÖGKJ Jahrestagung am 24.09.2020 um 17.30

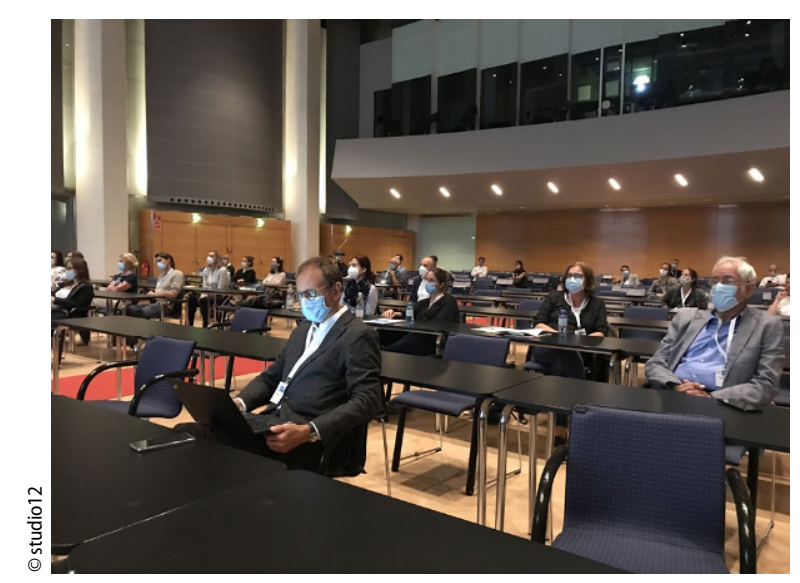

Abb. 7 - Parallelsitzung Saal Innsbruck, Kongresshaus Innsbruck, am 25.09.2020

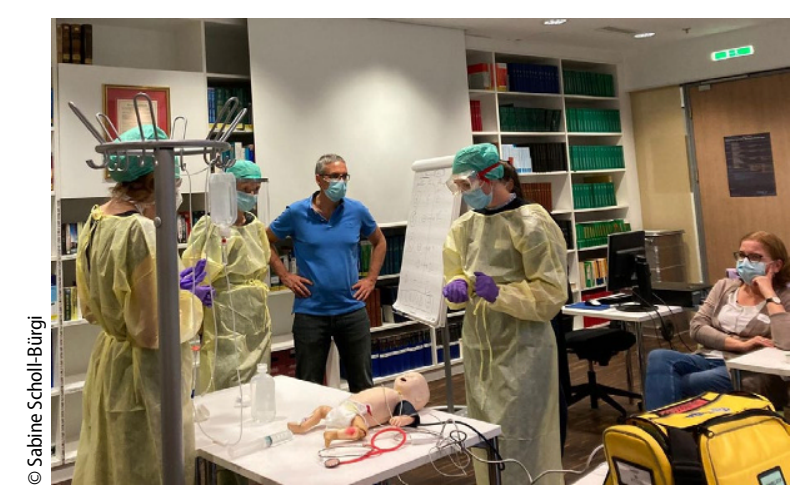

Abb. 8 A Workshop Kindernotfallmedizin drei Wochen in Innsbruck ausgestellt, bevor sie nach Südtirol weitertransportiert wurde. Weitere Informationen dazu siehe Mitteilungen der ÖGKJ in der Ausgabe 10-2020 der Monatsschrift Kinderheilkunde, https:// doi.org/10.1007/s00112-020-01026-3.

Von 19.00 bis 21.30 erlebten die an dem Abend 150 Teilnehmerinnen und Teilnehmer den Posterwalk, bei dem ein guter Austausch zwischen Jung und Alt, Ost und West, stationärem und niedergelassenen Bereich, ... stattfand immer unter Einhaltung der COVID-19 Präventionsmaßnahmen.

Auch die weiteren beiden Tage verliefen in einer ruhigen, sehr offenen, herzlichen Atmosphäre (• Abb. 7 und 8). Die insgesamt 400 Teilnehmerinnen und Teilnehmer betonten wiederholt, wie sehr sie das Wiedersehen mit Kolleginnen und Kollegen sowie den fachlich persönlichen Austausch vermisst haben und drückten ihre Dankbarkeit für das Stattfinden der Jahrestagung in diesem Rahmen aus.

Auch die Kolleginnen und Kollegen, die über Live Stream dabei waren, meldeten zurück, dass sie zwar betrübt seien, nicht persönlich anwesend sein zu können, aber dennoch dankbar seien, ein Teil des Ganzen zu sein und den Vorträgen folgen zu können. Insgesamt waren beim Live Stream knapp über 200 Zugriffe ersichtlich.

Ebenfalls erfolgreich fanden im Vorfeld der Tagung der Facharzt-Prüfungs-Vorbereitungskurs statt (Mittwochnachmittags und Donnerstagvormittags) und nach Abschluss am Samstagnachmittag zwei Workshops mit jeweils 20 Teilnehmern statt (Lungenfunktion, Leitung Anna Zschocke, Innsbruck, und Kindernotfallmedizin, Uwe Klingkowski, Innsbruck) (• Abb. 8).

Wir konnten nachvollziehen, dass die im Vorfeld generell aktuell verfügbaren Informationen über die Situation in Tirol/Innsbruck z.T. schwer einzuordnen waren, und auch, dass nicht alle mit der Situation „Abhaltung der Jahrestagung" gleich umgegangen wären, wie wir es zu tun entschieden haben. In dem Bewusstsein, dass die heurige Jahrestagung verständlicherweise anders sein würde als Tagungen zuvor, haben wir versucht, unsere Motivation und unseren Zugang zu der jeweiligen Situation zu erläutern.

Wir bedanken uns recht herzlich bei allen, die dazu beigetragen haben, dass dieses Pilotprojekt erfolgreich abgeschlossen werden konnte! Wir haben uns über jede(n) sehr gefreut, die/der unsere Philosophie mitgetragen hat und bei dieser Tagung anwesend war. Selbstverständlich haben wir auch Verständnis für jene, die die Situation anders gesehen haben und nicht bei der Tagung anwesend sein konnten.

Auch wenn die Industriepartner üblicherweise nicht in dem Jahrestagungsbericht erwähnt werden, möchten wir das heuer bewusst tun. Wir danken allen Firmensponsoren, die diese Jahrestagung und uns auch bei bereits im Vorfeld bekannter reduzierter Teilnehmerzahl durch alle Widrigkeiten in der Zeit der Vorbereitung verlässlich unterstützt haben! Das war nicht selbstverständlich - und wir haben es sehr geschätzt!

Und ... - wir freuen uns auf die Zeiten, wo wir uns alle wieder gemeinsam treffen können!

58. Jahrestagung der ÖGKJ, 24.09. bis 26.09.2020, Innsbruck Congress

\section{Verfasserinnen: \\ Sabine Scholl-Bürgi, Innsbruck Daniela Karall, Innsbruck}

Hinweis des Verlags. Der Verlag bleibt in Hinblick auf geografische Zuordnungen und Gebietsbezeichnungen in veröffentlichten Karten und Institutsadressen neutral.

Paediatr. Paedolog. 2020 · 55:316-319 https://doi.org/10.1007/s00608020-00850-w

c) Springer-Verlag GmbH Austria, ein Teil von Springer Nature 2020 
Hier steht eine Anzeige.

Springer 